\title{
Subpicosecond carrier transport in GaAs surface-space-charge fields
}

\author{
T. Dekorsy, T. Pfeifer, W. Kütt, and H. Kurz \\ Institut für Halbleitertechnik, Rheinisch Westfälische Technische Hochschule Aachen, \\ Sommerfeldstrasse 24, D-5100 Aachen, Germany
}

\begin{abstract}
Above-band-gap pulsed optical excitation of electron-hole pairs within the surface-space-charge region of semiconductors alters the surface-space-charge field via free-carrier transport. We report on the direct observation of this ultrafast transient screening and the associated charge-carrier transport by applying reflective electro-optic sampling (REOS) with subpicosecond time resolution to (100)oriented GaAs surfaces. The REOS measurements performed under different initial surface field conditions and various optical excitation densities are compared to numerical simulations of hotcarrier transport, including the calculation of the optical response. The simulations, which are based on a simple drift-diffusion model for optically excited electron-hole pairs, are in quantitative agreement with the experiment. The strength and sign of the static built-in field can be determined and the carrier drift velocities can be derived on a subpicosecond time scale.
\end{abstract}

\section{INTRODUCTION}

The transport dynamics of optically generated free electron-hole pairs in semiconductors and the associated screening of electrical fields are of great importance for the performance of high speed optoelectronic devices. Clear data on hot-electron dynamics are extremely important for a better understanding of the nonequilibrium phenomena involved, such as high-field transport, velocity overshoot, and ballistic transport.

Time-resolved optical techniques using femtosecond laser pulses are a unique tool for the determination of carrier dynamics on a subpicosecond time scale. The increasing temporal resolution to only some ten femtoseconds allows the time-resolved observation of dissipation processes such as carrier-carrier, carrier-phonon, and intervalley scattering. Recently, experiments with ultrashort laser pulses have been performed for studies of subpicosecond carrier transport dynamics. In these experiments the temporal evolution of average carrier transport parameters such as the drift velocity ${ }^{1}$ or the electron mobility ${ }^{2,3}$ have been investigated.

Reflective electro-optic sampling (REOS) is ideally suited for the study of transient field changes after optical excitation of free electron-hole pairs in surface-spacecharge fields. ${ }^{4}$ At bare semiconductor surfaces a static built-in electric field exists due to the pinning of the Fermi level at the energetic position of charged surface states. ${ }^{5}$ This effect leads to band bending and the buildup of a surface-space-charge region. After optical excitation, electron-hole separation due to field-induced carrier drift currents rapidly screens the surface field. This fast depolarization of the initial surface field is able to launch coherent LO phonons as clearly observed in previous experiments. ${ }^{6}$

The REOS signal is a direct measure of the temporal modification of the surface-space-charge field.
Anisotropic changes in the field-dependent part of the refractive index are detected via the linear electro-optic effect. Isotropic changes in the refractive index due to temporarily varying optical interband and intraband transition rates, i.e., changes in $\epsilon_{1}$, are canceled by using the appropriate probe geometry (Sec. II). It should be noted that carrier separation within the surface-space-charge region in turn leads to changes in $\epsilon_{1}$, which has to be considered in transient reflectivity measurements. ${ }^{7}$

REOS signals from (100)-orientated GaAs change sign when going from $n$ - to $p$-type GaAs due to the sign reversal of the band bending for the different doping types. ${ }^{5}$ The variation of the laser intensity shows a nonlinear dependence of the signal amplitudes to the excited carrier densities, which can be understood qualitatively in terms of transport theory.

In order to get a more detailed understanding of the measurements we performed numerical simulations of free charge-carrier transport pertinent to our experimental conditions. These simulations are based on a standard drift-diffusion equation (DDE) for electrons and holes. The DDE is the most simple formulation of the Boltzmann transport equation (BTE) and can be derived in the relaxation time approximation. ${ }^{8}$ Therefore the carrier distributions are treated as instantaneously thermalized and are described by heated Maxwell-Boltzmann functions with associated average transport parameters.

From the calculated spatial carrier distributions the electric field is self-consistently determined by solving Poisson's equation with appropriate boundary conditions. These boundary conditions include band bending due to Fermi-level pinning at the bare semiconductor surface. The electro-optic response is determined via an optical transfer-matrix method (TMM) (see Sec. III).

In Sec. IV we describe the results of simulation, e.g., the dependence of the calculated REOS signal on different carrier densities, different initial surface fields, and 
depletion region widths. The measured signals can be interpreted quantitatively in terms of incoherent carrier transport and associated transient screening lengths. It is not necessary to take into consideration a coherent, instantaneous nonlinear polarization as recently proposed by Chuang et $a .^{9}$ for the explanation of $\mathrm{THz}$ emission experiments.

Adjusting the simulation results by a parameter fit to the experimental curves allows the determination of the initial surface electric field and of electron and hole drift velocities under high field conditions on a subpicosecond time scale.

\section{REOS AT (100)-ORIENTED GaAs}

In the electro-optic sampling technique a pump and a probe beam, both derived from a colliding-pulse mode-locked (CPM) ring dye laser, are focused nearly collinearly onto a (100) sample. Pump and probe beams are polarized perpendicular to each other, while the probe beam is oriented along the [001] crystal direction [Fig. 1(b)]. The reflected probe beam is split into its orthogonal components $R_{\perp}$ and $R_{\|}$via a polarizing beam splitter, where the $\|$ orientation is taken to be along the [0힐 crystal axis [Fig. 1(a)]. Each probe beam component is detected with large area silicon pin photodiodes. The amplified difference signal $\Delta R=R_{\perp}-R_{\|}$ is recorded with a $4-\mathrm{MHz}$ analog-to-digital converter and further averaged with a high performance computer unit. The temporal resolution is achieved via a time-calibrated retroreflector mounted onto a shaker, thus varying the pump to probe time delay at a frequency of $50-100 \mathrm{~Hz}$ ("fast scan"). This technique allows fast averaging over $10^{5}$ scans in approximately $10 \mathrm{~min}$ providing a resolution of $\Delta R \sim 10^{-7}$ in most cases. ${ }^{10}$

The difference of the refractive index along the $[0 \overline{1} \overline{1}]$ and [01ㅣ crystal orientation is linearly proportional to the electric field along $z=[100]$ :

a)

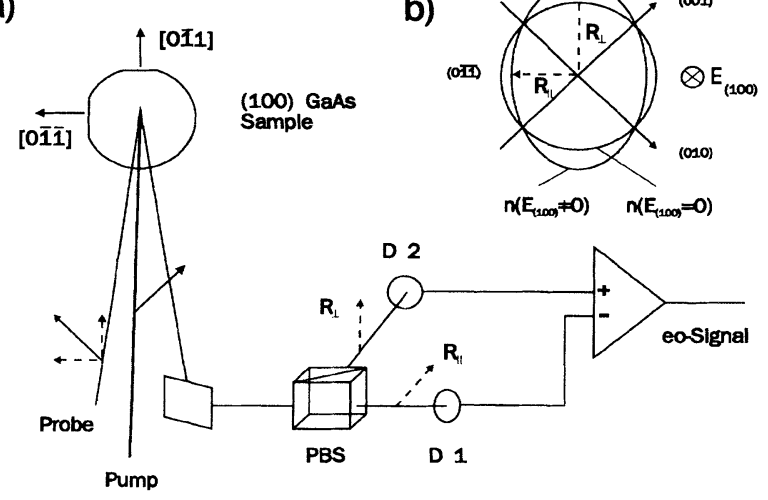

FIG. 1. Experimental REOS setup. (a) Pump-probe geometry with electro-optic sensitive detection (PBS polarizing beam splitter; $D 1, D 2$ detectors). (b) Optical indicatrix of the (100)-oriented GaAs surface in the case of zero and nonzero longitudinal electric field. $R_{\perp}$ and $R_{\|}$are the split reflected components of the probe beam.

$$
n_{\perp}-n_{\|}=n_{0}^{3} r_{41} E_{z}
$$

where $n_{0}$ is the isotropic refractive index, $r_{41}$ is the electro-optic coefficient of GaAs $\left[1.6 \times 10^{-10} \mathrm{~cm} / \mathrm{V}\right.$ (Ref. 11)], and $E_{z}$ is the longitudinal surface field. Thus the detected reflectivity changes $\Delta R$ associated with the electro-optic index changes are linearly proportional to changes in the longitudinal electric field $E_{z}$, when higher order terms in the electric field are neglected. Relative signal changes $\Delta R / R_{0}$ are obtained by dividing the measured signals by the unperturbed reflectivity $R_{0}$.

First subpicosecond time-resolved REOS measurements performed by Min and Miller have been used to study the vertical electron-hole transport in (100) GaAs surface-space-charge fields with a time resolution of approximately $700 \mathrm{fs}^{4}$ With an improved temporal resolution and the employment of "fast scan" detection techniques we are able to measure the electro-optic part of the reflectivity with high sensitivity. ${ }^{10}$ Signal oscillations due to coherently generated LO phonons are observed in this material system by this technique. ${ }^{6}$

To demonstrate the REOS sensitivity towards electric field changes, we performed experiments on GaAs Schottky junctions. The static built-in fields are varied by an externally applied voltage. For investigation of the field dependence of the screening indium-tin-oxide (ITO) Schottky contacts on $n$-type GaAs $\left(N_{D}=3 \times 10^{17} \mathrm{~cm}^{-3}\right)$ have been prepared. ITO contacts provide the advantage of high transparency to visible light and low electric resistivity. Optical measurements at different applied voltages can be carried out without disturbing contributions from the contact itself. The REOS signals at reverse bias voltages from $0 \mathrm{~V}$ to $-1.0 \mathrm{~V}$ are presented in Fig. 2. At zero externally applied voltage the signal exhibits an initial fast rise within $200 \mathrm{fs}$ followed by a slower one up to 1 ps. Both parts result from the transport of the photoexcited carriers and the associated decrease in the surface field. For longer times the signal remains at a quasiequilibrium level of $2 \times 10^{-4} \Delta R / R_{0}$. The equilibrium level rises with increasing reverse bias due to the increase in depletion width and in $E_{z}$ changes. The minute oscillations are coherently generated LO phonons. The dependence of the amplitude of the coherent oscillations on the initial field changes will be published elsewhere. ${ }^{12}$

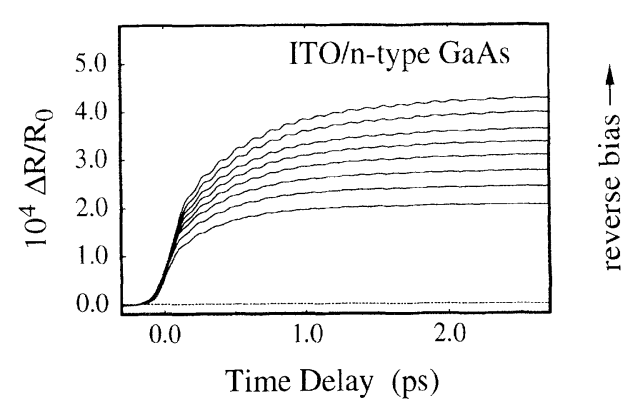

FIG. 2. REOS measurement on the ITO-GaAs transparent Schottky contact at different reversed bias voltages applied $(0$ to $-1 \mathrm{~V})$. 


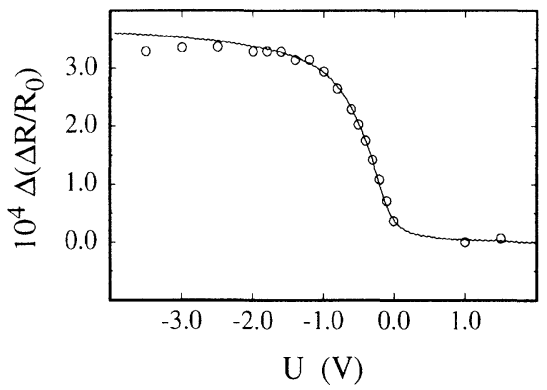

FIG. 3. Comparison between cw HeNe electro-optic reflectivity changes (solid line) under voltage modulation and time-resolved data taken from Fig. 2 at a time delay of $\sim 3$ ps (open circles).

In order to prove that the measured changes result from electro-optic effects, we performed one further experiment on the same sample with a $\mathrm{cw} \mathrm{HeNe}$ laser. By modulating the bias voltage applied to the ITOGaAs junction the electro-optic reflectivity changes of the polarized HeNe beam $(2 \mathrm{eV})$ are detected in the same probe geometry as in the CPM experiments. The relative electro-optic reflectivity changes of the voltagemodulation experiment $[\Delta R(U)-\Delta R(U=0 \mathrm{~V})] / R_{0}$ are compared to the relative values from the REOS curves from Fig. 2 at $3 \mathrm{ps}[\Delta R(3 \mathrm{ps}, U)-\Delta R(3 \mathrm{ps}, U=0 \mathrm{~V})] / R_{0}$ (Fig. 3). In the cw experiment the steady-state builtin field is measured as a function of applied voltage at low carrier injection densities, whereas the time-resolved measurement determines the deviation from the steadystate value due to screening. As the comparison clearly shows, the two signals are quantitatively equal. The differences in the two signals at reverse bias $>2.5 \mathrm{~V}$ result from the fact that the REOS signals at 3 ps still have not reached their quasiequilibrium values. However, this comparison demonstrates clearly the electro-optic nature of the transient REOS signals. Contributions to the REOS signals through changes in the refractive index due to free carriers are negligible.

In $F$ ig. 4 the REOS signals of $n$-type, $p$-type, and in-

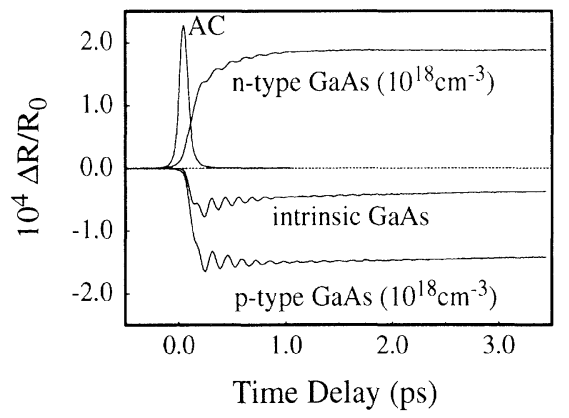

FIG. 4. REOS signal for $n$ - and $p$-doped material at a carrier density of $3 \times 10^{18} \mathrm{~cm}^{-3}$. The doping density for the $n$ and $p$ sample is $1 \times 10^{18} \mathrm{~cm}^{-3}$, while the intrinsic sample is background $p$ doped. AC denotes the autocorrelation of the pump pulse.

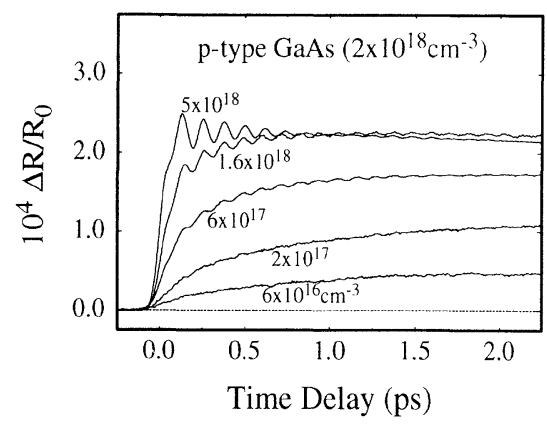

FIG. 5. REOS signal for $p$-type GaAs $(N=2 \times$ $\left.10^{18} \mathrm{~cm}^{-3}\right)$. The changes in the carrier densities cover two orders of magnitude $\left(6 \times 10^{16}\right.$ to $\left.5 \times 10^{18} \mathrm{~cm}^{-3}\right)$.

trinsic (100) GaAs samples are compared at carrier densities of $3 \times 10^{18} \mathrm{~cm}^{-3}$. The zero time delay is determined via two-photon absorption (TPA) in a thin GaP film. All REOS signatures exhibit an initial fast field change within the temporal width of the pump pulse. Periodic oscillations due to coherent LO phonons are superimposed.

The magnitude of REOS change after several picoseconds can be used as a direct probe of the initial surface field. For a quantitative analysis of the REOS data numerical simulations of transient field distributions and screening lengths are essential. Since the surface field $E_{z}(t)$ is spatially inhomogeneous within the absorption length of the probe beam (Sec. III), special methods for the calculation of the optical response have to be applied.

An obvious sign reversal occurs when going from $n$ type to $p$-type GaAs, since the electric surface field changes sign due to upwards band bending for $n$-type and downwards band bending for $p$-type GaAs. ${ }^{5}$ The negative sign for the nominally intrinsic sample results from background $p$ doping.

Another essential experiment concerns the dependence of the sreening dynamics on the optically injected carrier density. The variation of the excitation density over two orders of magnitude from $6 \times 10^{16} \mathrm{~cm}^{-3}$ to $5 \times 10^{18} \mathrm{~cm}^{-3}$ performed on $p$-doped GaAs $\left(N_{A}=2 \times 10^{10} \mathrm{~cm}^{-3}\right)$ reveals large effects on the screening of the initial surface field as demonstrated in Fig. 5. At low density only partial screening occurs and the dynamics of $E_{z}$ is determined by drift and diffusion. At high densities, however, complete screening occurs already during the excitation pulse, resulting in an extremely fast change of polarization, fast enough to launch coherent longitudinal vibrations.

\section{NUMERICAL SIMULATIONS}

Recently, various approaches for the modeling of shortpulsed laser excitation of bare semiconductor surfaces with regard to the temporal evolution of the built-in surface field have been published. Monte Carlo calculations for similar conditions as in our experiments consider the potential drop across the sample resulting from screening of the built-in field. ${ }^{13} \mathrm{~A}$ hydrodynamic description has 
been worked out for the electron-hole vertical transport in space-charge fields on a picosecond time scale. ${ }^{14}$ The influence of quantum-mechanical boundary conditions on transient screening of surface-space-charge fields has been treated using a real-space density-matrix formalism. ${ }^{15}$

The numerical simulations for the present study are based on a standard DDE for thermalized carrier distributions. The photoinjected electron-hole pairs are coupled to an electric field calculated via Poisson's equation in a self-consistent way. The validity of a DDE as it can be derived from the BTE in the relaxation time approximation depends on scattering rates. Detailed studies of carrier realaxation on a subpicosecond time scale have shown that ultrafast thermalization of hot photoexcited carriers via carrier-carrier scattering occurs within $100 \mathrm{fs}$ and less for excitation densities above $1 \times 10^{17} \mathrm{~cm}^{-3} \cdot 16,17$ Particularly strong intervalley scattering (IVT) implies extremely short impulse relaxation times and low electron mobilities of the hot electrons. ${ }^{3,18}$ Thus a driftdiffusion ansatz is applicable even on a subpicosecond time scale, although a number of rigid approximations has to be accepted. ${ }^{19}$

The starting point for the numerical simulations is the surface-space-charge field of free GaAs surfaces. The maximum electric surface field $E_{s}(z=0)$ and the depletion width $w$ are calculated from the well known equations: ${ }^{20}$

$E_{s}(z=0)=\left(\frac{2 q N_{d} \Phi_{b}}{\epsilon_{0} \epsilon_{s}}\right)^{1 / 2}$ and $w=\left(\frac{2 \epsilon_{0} \epsilon_{s} \Phi_{b}}{q N_{d}}\right)^{1 / 2}$

where $N_{d}$ is the doping density, $\epsilon_{0}$ is the dielectric permittivity, $\epsilon_{s}$ is the static dielectric constant, and $\Phi_{b}$ is the band bending at the samples surface. The band bending is a result of Fermi-level pinning at the midgap energetic level of charged surface states on (100) GaAs. ${ }^{5}$ In the Schottky model the electric field decreases linearly to zero within $w$. Deviations of Eq. (2) are associated with band bending without Fermi-level pinning as in the case of low surface charge densities. ${ }^{21}$

The basic one-dimensional DDE is solved for electrons and holes,

$$
\begin{aligned}
\frac{\partial N_{i}(z, t)}{\partial t}= & G(z, t)+\frac{\partial}{\partial z}\left\{D_{i}(z, t) \frac{\partial N_{i}(z, t)}{\partial z}\right\} \\
& \pm \frac{\partial}{\partial z}\left\{\mu_{i}(z, t) E(z, t) N_{i}(z, t)\right\}
\end{aligned}
$$

where $N_{i}$ is the carrier concentration $(i=e, h) . G(z, t)$ is the optical generation term, which is calculated from the laser intensity $I(t)$, the absorption coefficient $\alpha$, and the reflectivity $R$ via $G=I(t) \alpha(1-R) e^{-\alpha z}$. The temporal profile of the intensity $I(t)$ is Gaussian with a full width at half maximum (FWHM) of $50 \mathrm{fs}$. The $\mu_{i}$ and $D_{i}$ in Eq. (3) are the carrier mobilities and diffusion coefficients, respectively. Since the surface fields are of the order of $10 \mathrm{kV} / \mathrm{cm}$, the mobility is not well defined in the high field region. We rather consider the drift velocity $v_{d, i}=\mu_{i}(E) E$ as the most important simulation parameter, which entirely determines the transport dynamics.

The diffusion coefficient is related to the nonequilibrium transport properties and the carrier temperature via a generalized Einstein relation:

$$
D_{i}=\frac{k_{B} T_{i}}{e} \frac{v_{d, i}}{E}
$$

where $T_{i}$ is the carrier temperature $(i=e, h)$. A distinction between the two carrier temperatures $T_{e}$ and $T_{h}$ has to be taken into consideration because of different excess energies of the optically generated carriers $\left(\Delta E_{e} \approx 400\right.$ $\mathrm{meV}, \Delta E_{h} \approx 170 \mathrm{meV}$ ) and different energy dissipation rates.

The transient carrier temperatures $T_{i}$ are taken from the solution of a system of rate equations for thermalized distributions after femtosecond optical excitation. ${ }^{22}$ This model essentially gives an electron temperature $T_{e}$, which rises during the pump pulse to about $2000 \mathrm{~K}$ with a temperature cutoff for the $\Gamma$-valley distribution due to intervalley scattering ( $L$ valley) within $200 \mathrm{fs}$. The temperature of the thermalized distribution then decays exponentially with a time constant of $\sim 3 \mathrm{ps}$, taking into account the repopulation of the $\Gamma$ valley due to the return of side-valley electrons. The maximum hole temperature $T_{h}$ is well below the electron temperature with respect to the lower hole excess energy. In addition, the energy dissipation of holes is substantially faster than for electrons, ${ }^{23}$ thus the transient hole temperature has a minor effect on the simulation results.

From Poisson's equation the field distribution is calculated after each numerical time step for the integration of Eq. (3),

$$
\frac{\partial E(z, t)}{\partial z}=\frac{q}{\epsilon \epsilon_{0}}\left[N_{h}(z, t)-N_{e}(z, t) \pm N(z)\right],
$$

where $N(z)$ is the density of ionized donors or acceptors within the depletion width $w$. A transient redistribution of the free carriers introduced from the background doping is not taken into account, i.e., the transport of photoinjected carriers is supposed to determine the screening dynamics entirely. The numerical integration scheme for Eq. (3) is similar to the Crank-Nichelson method. ${ }^{24}$ The simulation volume covers a distance of twice the penetration depth $\alpha^{-1}$, where $\alpha$ is the absorption coefficient for GaAs at $620 \mathrm{~nm}\left(\alpha=4.3 \times 10^{4} \mathrm{~cm}^{-1}\right)$.

The boundary conditions for electron and hole currents are chosen to maintain conservation of particles, i.e., no charge carriers vanish in either the left $(z=0)$ or the right boundary $(z=L)$. No surface recombination is taken into account, although it can dominate surface dynamics on a picosecond time scale. ${ }^{25}$ The boundary condition for the electric field $E_{s}(z=0)$ is kept constant since changes in the charged surface states are neglected on this time scale. At $z=L$ the electric field is supposed to be zero.

The electro-optic response is calculated from the transient field distributions via the optical transfer-matrix method (TMM) ${ }^{26}$ The use of the TMM is essential because of large field gradients on length scales shorter than 
the absorption length. The TMM solves the electromagnetic wave equations in a layered system with varying optical coefficients $n(z, t)$ and $k(z, t)$ for each layer. The temporal and spatial changes in $n(z, t)$ are of electrooptic origin and are calculated from Eq. (1), while changes in the absorption coefficient are disregarded. The difference between the reflectivities for each polarization $R_{\perp, \|}$ is divided by the unperturbed reflectivity $R_{0}$. The use of the TMM enables a direct comparison between the calculated response and the experimental data. Additionally, the calculated response is convolved with a Gaussian probe pulse (FWHM of $50 \mathrm{fs}$ ).

\section{SIMULATION RESULTS AND DISCUSSION}

Simulations were carried out for the experimental conditions of various excitation densities $\left(1 \times 10^{16} \mathrm{~cm}^{-3}\right.$ to $5 \times 10^{18} \mathrm{~cm}^{-3}$ ) and samples of different doping densities $\left(1 \times 10^{16} \mathrm{~cm}^{-3}\right.$ to $\left.1 \times 10^{18} \mathrm{~cm}^{-3}\right)$. For this range of doping densities the associated surface field strengths and depletion widths vary from 40 to $400 \mathrm{kV} / \mathrm{cm}$ and from 300 to $30 \mathrm{~nm}$, respectively.

The simulation results for an $n$-doped sample $(3 \times$ $\left.10^{17} \mathrm{~cm}^{-3}\right)$ at an excitation density of $5 \times 10^{17} \mathrm{~cm}^{-3}$ are shown in Fig. 6. The simulations parameters are

$$
\begin{aligned}
\tau_{\mathrm{FWHM}} & =50 \mathrm{fs}, \\
\alpha & =4.3 \times 10^{4} \mathrm{~cm}^{-1}, \\
N_{D} & =3 \times 10^{17} \mathrm{~cm}^{-3}, \\
|E(z=0)| & =235 \mathrm{kV} / \mathrm{cm}, \\
w & =43 \mathrm{~nm}, \\
N_{\text {exc }} & =5 \times 10^{17} \mathrm{~cm}^{-3}, \\
v_{d, e} & =v_{d, h}=5 \times 10^{6} \mathrm{~cm} / \mathrm{s} .
\end{aligned}
$$

The drift velocities are restricted for both electrons and holes to $5 \times 10^{6} \mathrm{~cm} / \mathrm{s}$ in the high field regime. The diffusion coefficient is calculated from Einstein's equation with the temperature model for thermalized carrier distributions.

Simulation results are presented in Fig. 6. The electron and hole distributions separate spatially within the surface-space-charge region [Fig. 6(a)]. The electrons are repelled from the surface, while the holes accumulate at $z=0$. Even at zero time delay, already a small separation of electrons (dashed line) and holes (solid line) is observed, with a difference maximum in the carrier densities $N_{e}-N_{h}$ at $z=0$ and $z=w$. This separation occurs during the first half of the pump pulse. At a time delay of $100 \mathrm{fs}$ a distinct carrier separation is established. The electrons are accumulated in a depth of $\sim 30 \mathrm{~nm}$. The holes, however, are swept towards the surface and reach a maximum value of $2.5 \times 10^{18} \mathrm{~cm}^{-3}$ at $z=0$. The carrier distribution in the case of negligible drift and diffusion (dotted line) is nearly homogeneous within the depletion width $\left(\alpha^{-1}>w\right)$. At longer time delays (not shown) the distributions reach a quasiequilibrium, which is governed by the balance between drift and diffusion currents. Deviations from this quasiequilibrium occur due to cooling of the carrier distributions. The transient temperature loss of the hot carriers leads to a reduced diffusion relative to the field drift, as given by the Einstein relation. A reduced electron diffusion leads to a sharply peaked electron distribution, screening the surface field more effectively. The ratio of the excitation density to conduction-band density of states $\left(N_{C}=4.7 \times 10^{17} \mathrm{~cm}^{-3}\right)$ affects the diffusion coefficient in the case of degeneracy. ${ }^{20}$

For even longer times bulk and surface recombination ${ }^{25}$ as well as changes in the surface-state occupation factors $^{21}$ have to be taken into account for the evaluation of the surface field.

The resulting transient field distributions are shown in
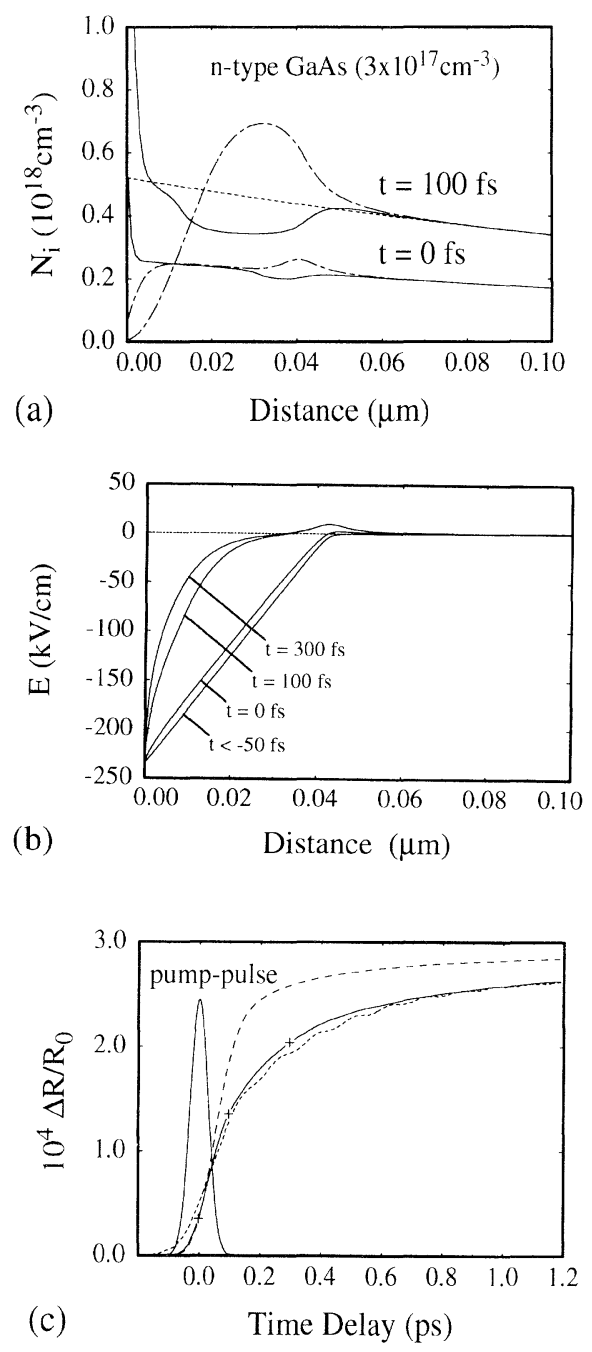

FIG. 6. Results from numerical calculations for an $n$ doped sample $\left(N_{D}=3 \times 10^{17} \mathrm{~cm}^{-3}, N_{\text {exc }}=5 \times 10^{17} \mathrm{~cm}^{-3}\right)$ : (a) Spatial carrier distributions at $t=0 \mathrm{fs}$ and $t=100 \mathrm{fs}$ for a FWHM 50-fs pump pulse (solid line: hole distribution; dashed line: electron distribution; dotted line: distribution without regarding carrier transport). (b) Transient electric field profiles with boundary condition $E(z=0)=-235$ $\mathrm{kV} / \mathrm{cm}$. (c) Calculated REOS signal (solid line), experimental data (dotted line), and simulation without hot carrier effects $\left(T_{e}=T_{h}=300 \mathrm{~K}\right)$ (dashed line). Times corresponding to (b) are marked with crosses. 
Fig. 6(b). At the maximum of the pump pulse at $t=0$ the initial field is already slightly decreased. At a time delay of $100 \mathrm{fs}$ the surface charges are screened at a distance of less than $w / 2$. At longer time delays the screening length is further decreased due to further redistribution of the charge carriers. The field and carrier distributions are similar to those obtained from Monte Carlo calculations by Zhou, Hsiang, and Miller, ${ }^{13}$ although there, the holes are kept immovable. The screening dynamics for both types of band bending are dominated by majority carriers, which are driven into the bulk, thus building up a very effective polarization. But even in $n$-type material the holes contribute to screening, since high field drift velocities of electrons and holes are comparable, as long as the satellite valleys ( $X, L$ valleys) are occupied. This contribution of minority carrier drift is clearly observed in Fig. 6(a).

The calculated electro-optic response is compared to the experimental data [Fig. 6(c)]. The data are convolved with the probe pulse intensity. The assumed Gaussian pump pulse intensity is also indicated. The crosses indicate the time delays, for which carrier and field distributions are given in Figs. 6(a) and 6(b). According to the optical detection mechanism field changes at the surface contribute stronger to the electro-optic response than those in a certain distance. Thus the REOS signal is not a linear function of the screening length. Regarding the uncertainty in the experimental conditions with respect to the correct excitation density and pulse shape the simulation fit the experimental data very well. The dashed line is calculated without regarding the influence of hotcarrier effects, i.e., the temperature of electrons and holes is kept constant at $300 \mathrm{~K}$. The effect of transient electron temperatures is a reduction of the fast initial screening due to the increased electron diffusion. When the carriers cool down to the lattice temperature $(t>3 \mathrm{ps})$, the influence of the carrier temperatures vanishes. In the case of dominant hole transport, this temperature effect is not observed, because of the lower hole temperature and their fast energy relaxation. ${ }^{23}$ This difference between $n$ - and $p$-type materials is clearly observed in the experimental curves in Fig. 2. Both, excitation and doping density, are the same there, but the signal rises slower within the first picosecond in the $n$-doped sample than in the $p$-doped one due to the hot-electron diffusion effect. This particular difference is observed for all $n$ - and $p$-type samples at direct comparison (equal doping and excitation density). This is a clear observation of a nonambipolar diffusion effect of hot electrons on a subpicosecond time scale.

Further numerical investigations have been carried out on the variation of the optically injected carrier density. Figure 7 shows the comparison between calculated and experimental REOS signals on $p$-doped GaAs $\left(2 \times 10^{18} \mathrm{~cm}^{-3}\right)$ within the first picosecond after excitation. The experimental data are well reproduced by the calculations with one set of parameters over a wide range of excitation densities. The simulations parameters are the same as before, except $|E(z=0)|=350 \mathrm{kV} / \mathrm{cm}$ and $w=30 \mathrm{~nm}$. The excitation densities in the calculation were adjusted to the experimental conditions. In contrast to the results for $n$-doped GaAs as considered

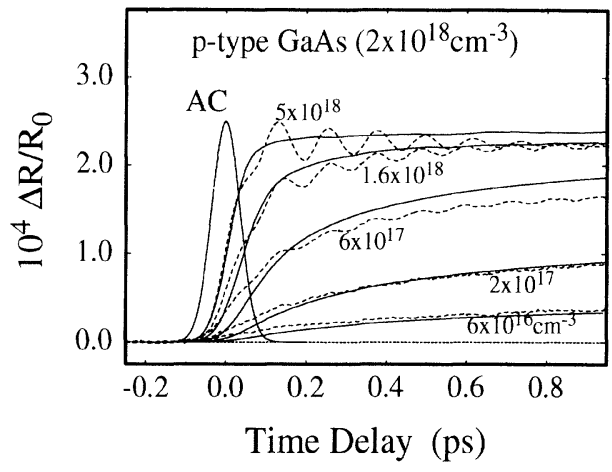

FIG. 7. Calculated REOS signals (solid lines) and experimental results (dashed lines) at different carrier densities in $p$-doped (100) GaAs $\left(2 \times 10^{18} \mathrm{~cm}^{-3}\right)$.

above, in the $p$-type case the holes are repelled from the positive charged surface while the electrons drift towards the surface. The drift velocities for electrons and holes are restricted to high field saturation values of $5 \times 10^{6}$ $\mathrm{cm} / \mathrm{s}$.

The observed changes in the screening dynamics with increasing excitation intensity are well reproduced by the simulation. They are also understood by the following, more intuitive explanation. The initial surface field is depolarized by the polarization which is built up from carrier separation

$$
\Delta P(t)=q N_{\mathrm{exc}}(t, z) \Delta z(t),
$$

where $\Delta z(t)$ is the spatial separation of electrons and holes and $\Delta P(t)$ is the associated polarization in the $z$ direction. At high excitation densities small $\Delta z$ are required for a fast and effective screening, while at low excitation densities the same value for $\Delta P(t)$ is achieved via carrier transport over longer distance $\Delta z(t)=v_{d, i} t$. All calculations fit well to the experimental data over two orders variation in excitation densities and different doping types and levels with drift velocities $v_{d, e}<1 \times 10^{7} \mathrm{~cm} / \mathrm{s}$ and $v_{d, h}=5 \times 10^{6} \mathrm{~cm} / \mathrm{s}$. This justifies the assumption that the screening dynamics is determined by saturated electron and hole drift velocities.

These drift velocities are of the order of steady-state drift velocities in GaAs. There is no velocity overshoot observed in these experiments, which is in agreement with the predictions from MC calculations for 2-eV excitation in high electric fields. ${ }^{27}$ The reason is the strong IVT of electrons excited with 2-eV photons, which results in a mean excess energy $(\sim 400 \mathrm{meV})$ above the $\Gamma-L$ energy splitting. Velocity overshoot may only be observed when the initial average electron energy is well below the average electron energy in the electric field. Additionally, when the surface field is screened rapidly ( $\sim 100 \mathrm{fs})$ at high excitation densities, the average electron energy is high above the steady-state value for screened electric fields.

It should be noted that the REOS experiments of this study are very closely related to $\mathrm{THz}$ radiation experiments performed recently on free III-V semiconductor surfaces. The time-varying currents responsible for the 
screening of the surface-space-charge field observed in the REOS experiments radiate electromagnetic waves of $\mathrm{THz}$ frequencies, which are detected via photoconducting antennas. ${ }^{28}$ However, these experiments differ from the REOS experiments in several aspects. The detection of the $\mathrm{THz}$ radiation monitors the second time derivative of the polarization near the surface, while we directly observe the longitudinal polarization changes by means of the electro-optic effect. In contrast to the REOS experiments, the $\mathrm{THz}$ experiments are not performed under normal incidence of the pump beam. Another difference concerns the time resolution, which is limited for the $\mathrm{THz}$ detection by the frequency response of the antennas to $\sim 3 \mathrm{THz}$.

A recent theory by Chuang et al. ${ }^{9}$ proposes a coherent optical rectification mechanism to be responsible for the generation of the $\mathrm{THz}$ radiation. The model is based on nonvertical transitions of electrons and holes in real space in the presence of a static electric field. This effect should lead to a coherent instantaneous depolarization of the initial surface field during the pump pulse, restricted to specific selection rules concerning the pump pulse polarization and the crystallographic axis of the sample. We observe a fast screening within the pump pulse duration at high excitation densities, which is entirely understood from classical, incoherent carrier transport. Additionally, the proposed pump polarization dependence of the coherent rectification mechanism is not observed in our electro-optic setup. However, a clear distinction between coherent and incoherent screening effects is somewhat artificial, since the coherent regime approaches the incoherent on a time scale of the dephasing time $T_{2}$. The generation of coherent LO phonons, which are generated via the fast initial surface-field changes, can be explained with the present calculations as well. ${ }^{29}$

The combination of REOS experiments with the presented simulations can be used as a tool for the quantitative determination of electric surface fields. They provide a direct measure of the initial surface field and have therefore some advantages compared with modulation spectroscopy methods such as electroreflectance, photoreflectance, or reflection difference spectroscopy. The REOS sensitivity to different doping densities may be of interest for the determination of lateral doping profiles. Changes in the surface electric field due to process induced defects ${ }^{30}$ or chemical passivation can be monitored, too.

\section{CONCLUSIONS}

We report on reflective electro-optic sampling experiments on (100) GaAs with a subpicosecond time resolution. The photoexcited electron-hole pairs are separated within the surface-space-charge field leading to an ultrafast screening of charged surface states. We have investigated the transport dynamics using a standard driftdiffusion model for the transport of free charge carriers. Although a drift-diffusion equation based on thermalized carrier distributions implies a number of rigid approximations, they explain successfully the observed transport dominated screening effects. The electro-optic response evaluated via the optical transfer-matrix method allows one to obtain the average transport parameters. The high field drift velocities of electrons and holes, and the initial surface field strength, are determined. A difference in the screening dynamics between $n$ - and $p$-type GaAs is explained by hot-electron diffusion.

Quantitative agreement between experiment and simulation has been achieved by assuming average drift velocities below $1 \times 10^{7} \mathrm{~cm} / \mathrm{s}$ for electrons and $5 \times 10^{6} \mathrm{~cm} / \mathrm{s}$ for holes at optical excitation densities between $6 \times$ $10^{16} \mathrm{~cm}^{-3}$ and $5 \times 10^{18} \mathrm{~cm}^{-3}$. A weak dependence of the drift velocities on the excitation density is observed only. This drift velocities do not exceed the steady-state saturation values in GaAs, i.e., there is no velocity overshoot observed even on a subpicosecond time scale. The suppression of velocity overshoot is attributed to strong intervalley scattering of the optically excited electrons with an excess energy above the $\Gamma-L$ valley energy splitting.

\section{ACKNOWLEDGMENTS}

Useful discussions with R. Scholz, H. Mikkelsen, and S. Hunsche are gratefully acknowledged. We like to thank A. Förster and H. Lüth (ISI, KFA Jülich) for supplying molecular-beam epitaxy samples and B. Görig (Humboldt University Berlin) for the ITO-GaAs sample. This work was entirely supported by the "Deutsche Forschungsgemeinschaft."
${ }^{1}$ C. V. Shank, R. L. Fork, B. I. Greene, F. K. Reinhart, and R. A. Logan, Appl. Phys. Lett. 38, 104 (1981).

${ }^{2}$ M. C. Nuss, D. H. Auston, and F. Capasso, Phys. Rev. Lett. 58, 2355 (1987).

${ }^{3}$ M. C. Nuss, in Ultrafast Phenomena VI, edited by T. Yajima, K. Yoshihara, C. B. Harris, and S. Shinoya (SpringerVerlag, Berlin, 1988).

${ }^{4}$ L. Min and R. J. D. Miller, Appl. Phys. Lett. 56, 524 (1990).

${ }^{5}$ W. E. Spicer, Z. Liliental-Weber, E. Weber, N. Newman, T. Kendelewicz, R. Cao, C. McCants, P. Mahowald, K. Miyano, and I. Lindau, J. Vac. Sci. Technol. B 6, 1245 (1988).
${ }^{6}$ G. C. Cho, W. Kütt, and H. Kurz, Phys. Rev. Lett. 65, 764 (1990).

${ }^{7}$ R. A. Cheville, W. B. Haynes, and N. J. Halas, Appl. Phys. Lett. 59, 1476 (1991).

${ }^{8}$ W. Hänsch, The Drift-Diffusion-Equation and Its Application in MOSFET Modeling, edited by S. Selberherr (Springer-Verlag, New York, 1991).

${ }^{9}$ S. L. Chuang, S. Schmitt-Rink, B. I. Green, P. E. Saeta, and A. J. Levi, Phys. Rev. B 68, 102 (1992).

${ }^{10}$ M. Strahnen, W. Kütt, and H. Kurz, in Proceedings of an International Conference on VME-bus in Research, edited by C. Eck (North-Holland, Amsterdam, 1988), p. 69. 
${ }^{11}$ A. Yarif, Introduction to Optical Electronics (Holt, Rinehart and Winston, New York, 1971).

${ }^{12}$ T. Pfeifer, T. Dekorsy, W. Kütt, and H. Kurz, Appl. Phys. A 55, 482 (1992).

${ }^{13}$ X. Zhou, T. Y. Hsiang, and R. J. D. Miller, J. Appl. Phys. 66, 3066 (1989).

${ }^{14}$ T. Held, T. Kuhn, and G. Mahler, Phys. Rev. B 44, 12873 (1991).

${ }^{15}$ R. Scholz and A. Stahl, Phys. Status Solidi B 168, 123 (1991).

${ }^{16}$ W. Z. Lin, R. W. Schoenlein, J. G. Fujimoto, and E. P. Ippen, IEEE J. Quantum Electron. QE-24, 267 (1988).

${ }^{17}$ W. H. Knox, D. S. Chemla, G. Livescu, J. E. Cunningham, and J. E. Henry, Phys. Rev. Lett. 61, 1290 (1988).

${ }^{18}$ E. Constant, in Hot Electron Transport in Semiconductors, edited by L. Reggiani (Springer-Verlag, Berlin, 1985).

${ }^{19}$ J. P. Nougier, J. C. Vaissiere, D. Gasquet, J. Zimmermann, and E. Constant, J. Appl. Phys. 52, 825 (1981).

${ }^{20}$ S. M. Sze, Physics of Semiconductor Devices, 2nd ed. (Wiley, New York, 1981).
${ }^{21}$ R. B. Darling, Phys. Rev. B 43, 4071 (1991).

${ }^{22}$ U. Lemmer, Diploma thesis, Rheinisch Westfälische Technische Hochschule Aachen, 1990.

${ }^{23}$ X. C. Zhou, K. Leo, and H. Kurz, Phys. Rev. B 45, 3886 (1992).

${ }^{24}$ W. H. Press, B. P. Flannery, S. A. Teukolsky, and W. T. Vetterling, Numerical Recipes in $C$ (Cambridge University Press, London, 1988).

${ }^{25}$ D. C. Marvin, S. M. Beck, J. E. Wessel, and J. G. Rollins, IEEE J. Quantum Electron. QE-25, 1064 (1989).

${ }^{26} \mathrm{M}$. Born and E. Wolf, Principles of Optics (Pergamon, Oxford, 1960).

${ }^{27}$ G. M. Wysin, D. L. Smith, and A. Redondo, Phys. Rev. B 38, 12514 (1988).

${ }^{28}$ X.-C. Zhang and D. H. Auston, J. Appl. Phys. 71, 326 (1992).

${ }^{29}$ W. Kütt, T. Pfeifer, T. Dekorsy, and H. Kurz, Int. Conf. Quantum Electron. Tech. Dig. Ser. 9, 312 (1992).

${ }^{30}$ W. Kütt, G.C. Cho, M. Strahnen, and H. Kurz, Appl. Surf. Sci. 50, 325 (1991). 